\title{
EXPRESSION OF GENETIC MARKERS OF ENDOTHELIAL PATHOLOGY IN PREGNANT WOMEN
}

\author{
Eugeny Yupatov ${ }^{1}$, Larisa Maltseva ${ }^{1}$, Olga Kravtsova², Tatiana Zefirova ${ }^{1}$ \\ 1 Obstetrics and Gynecology, Kazan State Medical Academy - Branch Campus of the Federal State Budgetary Educational \\ Institution of Further Professional Education «Russian Medical Academy of Continuing Professional Education» of the Ministry \\ of Healthcare of the Russian Federatio, Kazan, Russia \\ 2 Institute of Fundamental Medicine, Kazan Federal University, Kazan, Russia
}

Problem statement. Pregnancy considers to be well-established risk factor for venous system disorders. The compound of cardio-vascular changes during pregnancy provides significant load on veins, which increases with gestational age and may be accompanied by venous endothelial damage. This in turn can lead to the development of perinatal complications associated with endothelial dysfunction. The detection of biochemical markers of venous endothelial disfunction may help early diagnosis of pregnancy complications and expand the possibilities of preventive therapy.

The goal: to study the features of expression of markers of endothelial vein dysfunction in women depending on pregnancy outcomes.

Methods: There were 100 pregnant women in whom the expression of adhesion molecules ICAM-1, VCAM-1 and hypoxia gene HIF 1 were determined in the dynamics of pregnancy. Analysis of gene expression was performed by real-time PCR using commercial probes and primers (Applied Biosystems, Hs00234077_m1) on a CFX96 thermocycler (BioRad, USA). The reference gene used gene ACTB (Hs01060665_g1). At the end of pregnancy, women who gave birth to children with pathology of the early neonatal period were identified. The analysis of ICAM-1, VCAM-1 and hypoxia gene HIF 1 was carried out depending on pregnancy outcomes for the fetus.

Results. In women who gave birth to children with complications of the early neonatal period the expression of the cell adhesion molecule ICAM1 is 6.1 times higher than in women who gave birth without complications. Such dynamics is observed in the expression of Hif1a gene - its expression is higher by 9.7 times in women who gave birth to children with pathology. VCAM 1 gene activity was not detected in any study group.

Conclusion. The study of the expression of adhesion molecules ICAM-1, VCAM-1 and hypoxia gene HIF 1 demonstrated 
promising value for predicting complications of the neonatal period 